Supplement of Earth Syst. Dynam., 11, 509-524, 2020

https://doi.org/10.5194/esd-11-509-2020-supplement

(c) Author(s) 2020. This work is distributed under

the Creative Commons Attribution 4.0 License.

(c) (1)

Earth System
Dynamics

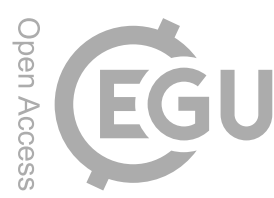

Supplement of

\title{
Eurasian autumn snow link to winter North Atlantic Oscillation is strongest for Arctic warming periods
}

Martin Wegmann et al.

Correspondence to: Martin Wegmann (martin.wegmann@awi.de)

The copyright of individual parts of the supplement might differ from the CC BY 4.0 License. 


\begin{tabular}{|c|c|c|c|c|c|c|}
\hline a) & 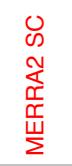 & 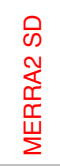 & 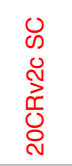 & $\begin{array}{l}\text { D } \\
\text { U } \\
\stackrel{N}{\mathbb{N}} \\
\text { d } \\
\text { D }\end{array}$ & 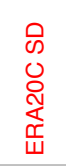 & 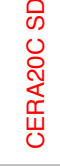 \\
\hline MERRA2 SC & 1 & 0.86 & 0.94 & 0.89 & 0.88 & 0.92 \\
\hline MERRA2 SD & 0.86 & 1 & 0.76 & 0.81 & 0.91 & 0.89 \\
\hline 20CRv2c SC & 0.94 & 0.76 & 1 & 0.92 & 0.76 & 0.81 \\
\hline 20CRv2c SD & 0.89 & 0.81 & 0.92 & 1 & 0.7 & 0.79 \\
\hline ERA20C SD & 0.88 & 0.91 & 0.76 & 0.7 & 1 & 0.93 \\
\hline CERA20C SD & 0.92 & 0.89 & 0.81 & 0.79 & 0.93 & 1 \\
\hline
\end{tabular}

\begin{tabular}{|c|c|c|c|c|c|}
\hline 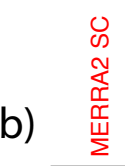 & 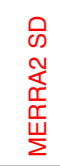 & 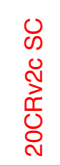 & 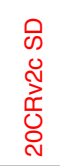 & 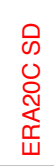 & 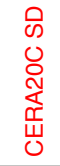 \\
\hline 1 & 0.85 & 0.87 & 0.83 & 0.91 & 0.91 \\
\hline 0.85 & 1 & 0.77 & 0.69 & 0.79 & 0.8 \\
\hline 0.87 & 0.77 & 1 & 0.94 & 0.77 & 0.77 \\
\hline 0.83 & 0.69 & 0.94 & 1 & 0.77 & 0.8 \\
\hline 0.91 & 0.79 & 0.77 & 0.77 & 1 & 0.94 \\
\hline 0.91 & 0.8 & 0.77 & 0.8 & 0.94 & 1 \\
\hline
\end{tabular}

3

4 Figure 1: Correlation of snow depth (SD) and snow cover (SC) indices for different reanalyses products for a) 5 October snow indices and b) November snow indices. The correlation coefficient is computed for the respective 6 shared time period among two products (see also Fig. 1). 

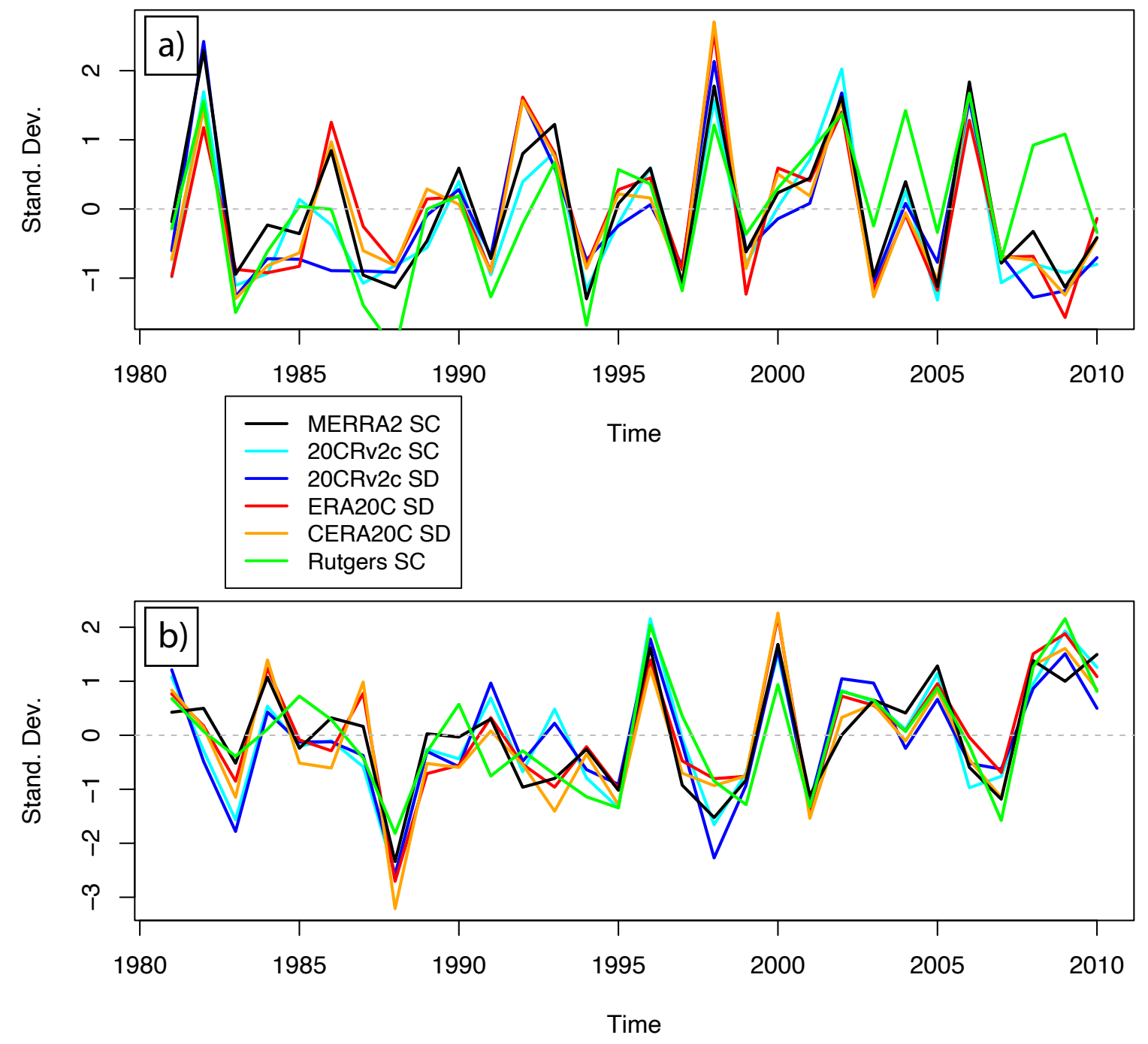

Figure 1: Comparison of a) October snow indices and b) November snow indices for the period 1981-2010 in multiple reanalysis products as well as the Rutgers snow laboratory satellite-based snow cover product (Robinson et al. 2012). 

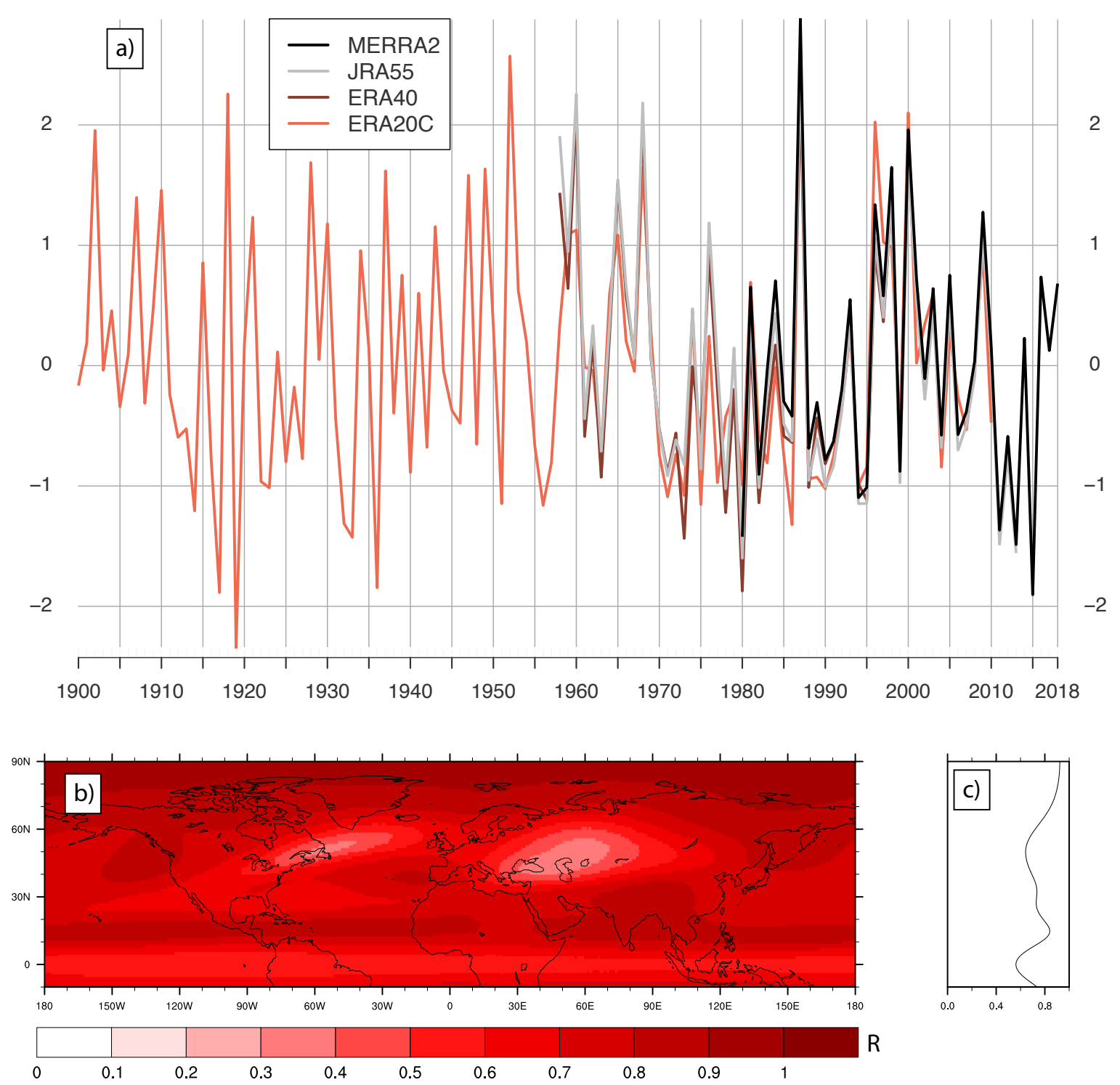

13

14 Figure 3: Evaluation of November December mean of polar cap $10 \mathrm{hPa}$ GPH in ERA20C. a) Field mean

15 November December $10 \mathrm{hPa}$ GPH normalized index comparison between MERRA2, JRA55, ERA40 and

16 ERA20C, b) correlation of November December $10 \mathrm{hPa}$ GPH anomalies between MERRA2 and ERA20C with c)

17 the zonal mean of the correlation coefficients. 

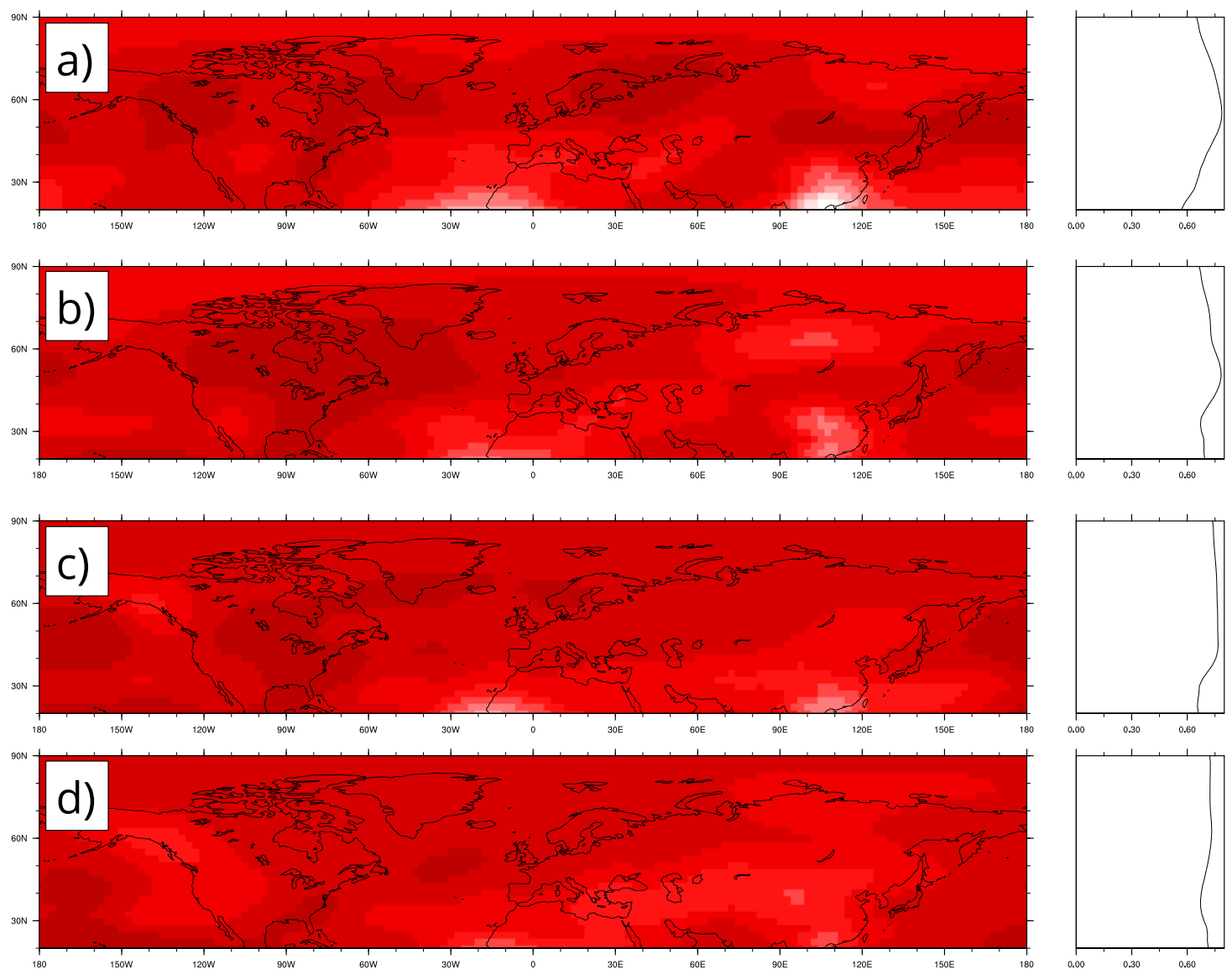

18

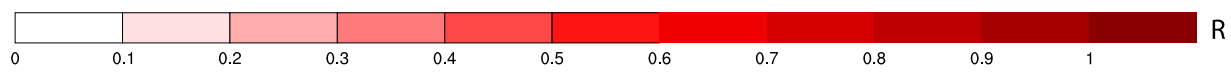

19 Figure 4: Correlation of $100 \mathrm{hPa}$ GPH anomalies between ERA20C and a merged product of JRA55 fields

20 (1958-2010) and statistically reconstructed monthly geopotential height fields for the period 1880-1957

21 (Griesser et al. 2010) for a) November, b) December, c) January and d) February for 1901-2010 
autocorrelation function Nov. 20CRv2c snow cover index

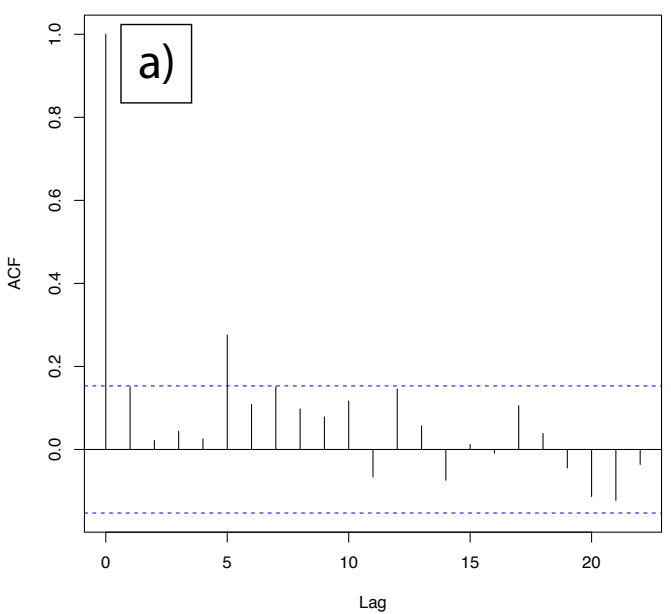

autocorrelation function Nov. ERA20C snow cover index

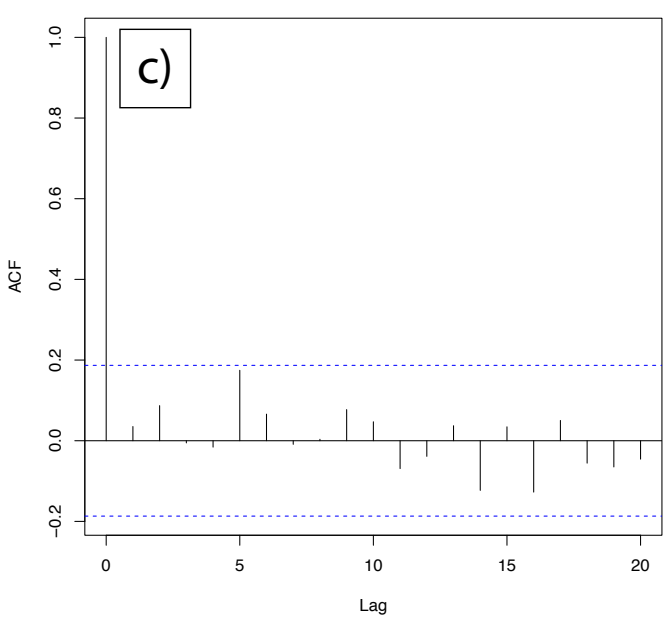

autocorrelation function Walsh et al. Nov. BKS sea ice index

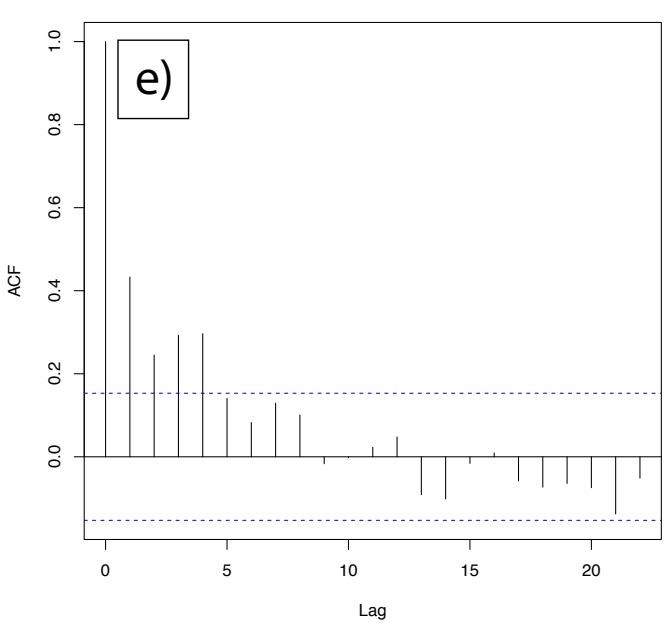

autocorrelation function Oct. 20CRv2c snow cover index

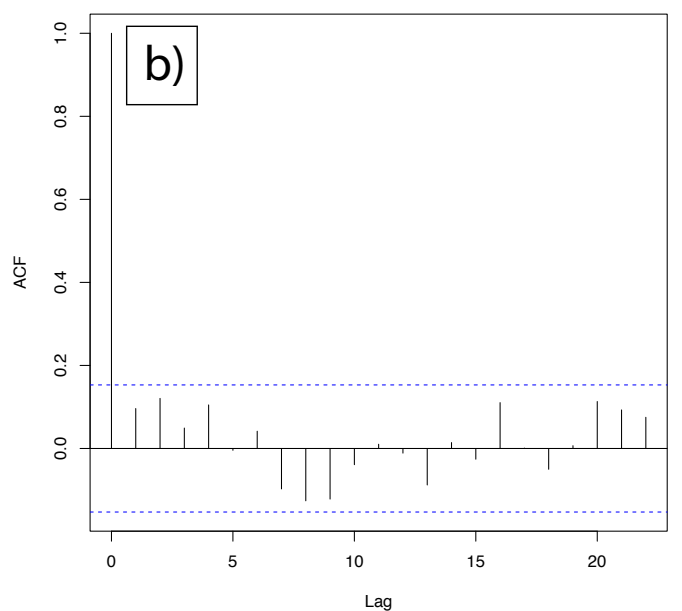

autocorrelation function Oct. ERA20C snow cover index

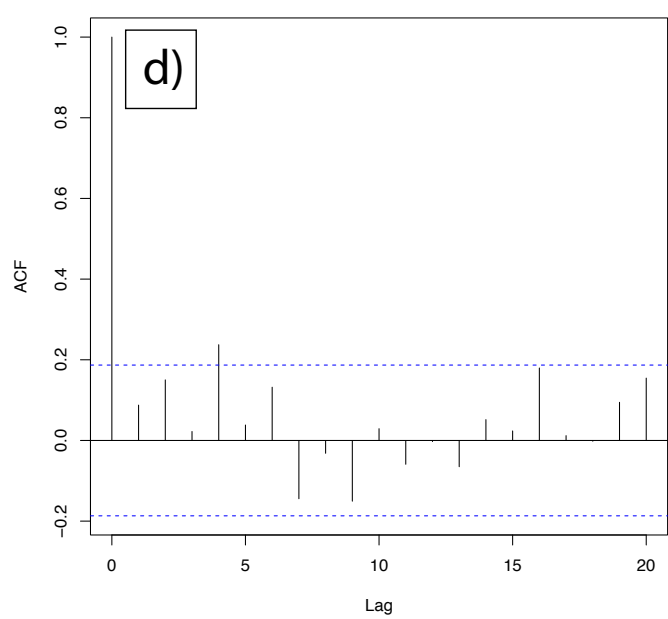

autocorrelation function ND ERA20C $10 \mathrm{hPa}$ polar cap index

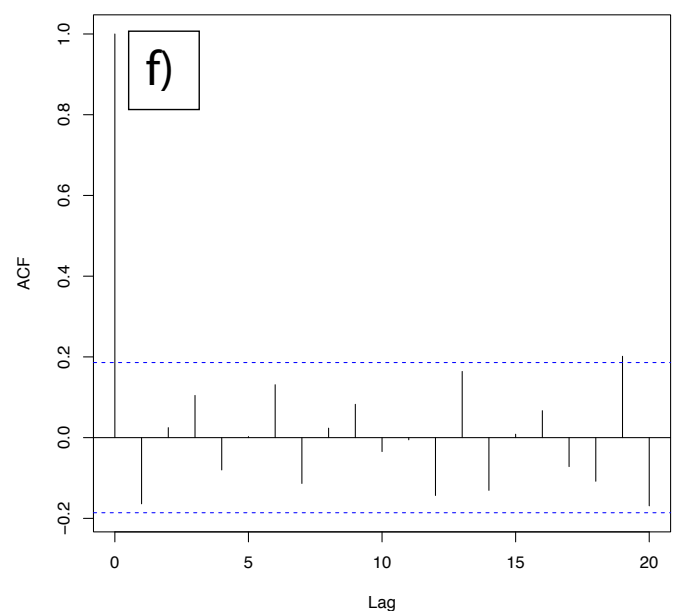

23 Figure 5: Autocorrelation functions up to lag 20 for a) the 20CRv2c November normalized detrended snow cover index, b) the 20CRv2c October normalized detrended snow cover index, c) the ERA20C November normalized detrended snow cover index, d) the ERA20C October normalized detrended snow cover index, e) the Walsh et al. November normalized detrended BKS sea ice index and f) the ERA20C November December mean normalized detrended 10hPa GPH index. 

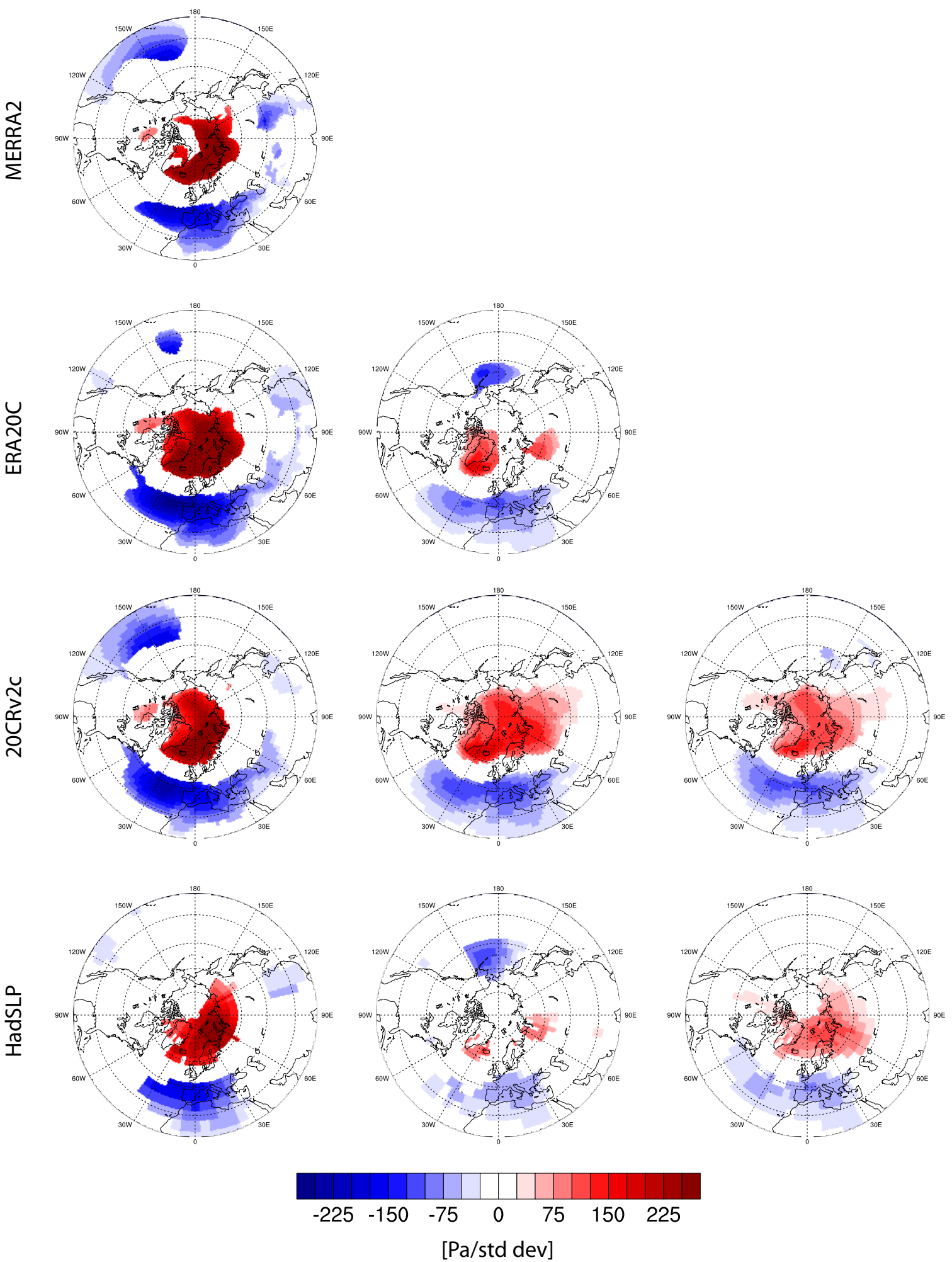

30 Figure 6: DJF sea level pressure [Pa/std dev] anomalies projected onto snow index in November MERRA2,

31 ERA20C, 20CRv2c and HadSLPr2 covering different time slices. 

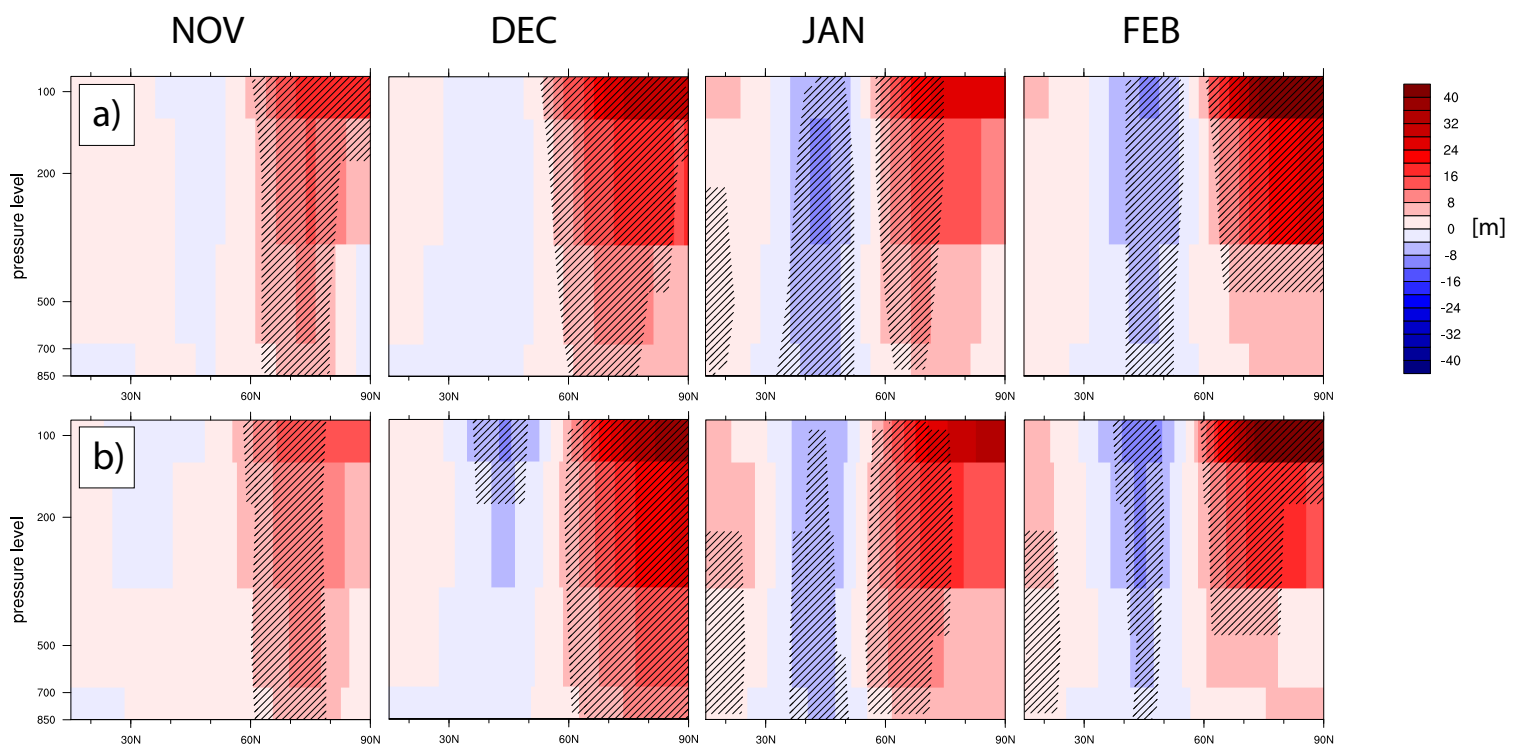

Figure 7: Zonal mean $\left(180^{\circ} \mathrm{E}-180^{\circ} \mathrm{W}, 15^{\circ} \mathrm{N}-90^{\circ} \mathrm{N}\right)$ geopotential height anomalies on 100, 200, 500 and $850 \mathrm{hPa}$ projected onto snow indices in November for a) a merged product of JRA55 fields (1958-2010) and statistically reconstructed monthly geopotential height fields for the period 1880-1957 (Griesser et al. 2010) and b) ERA20C covering 1901-2010. Shading indicates 95\% significance level. 


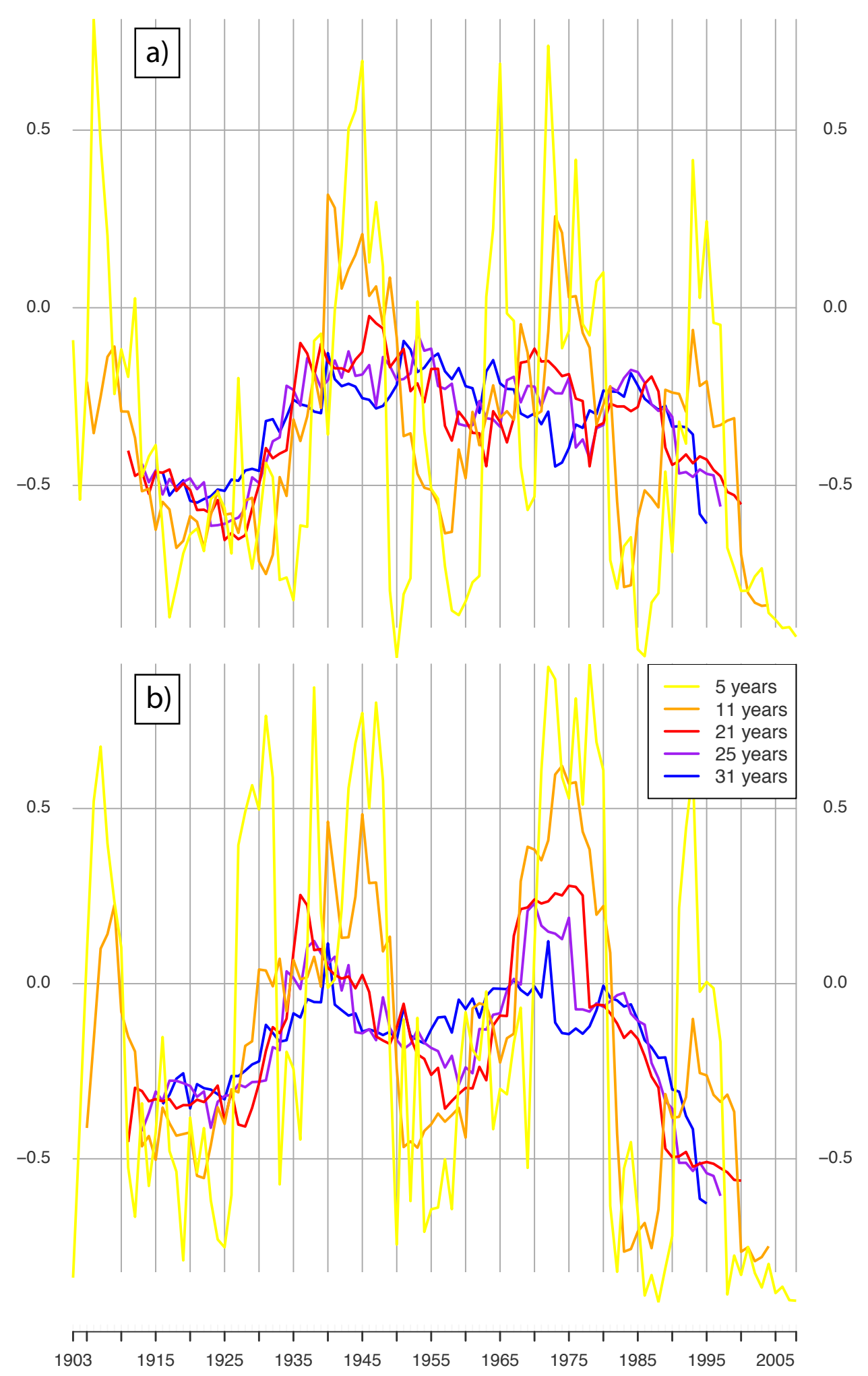

40 Figure 8: Comparison of different running correlation [R] windows between a) 20CRv2c November snow index

41 and DJF NAO and b) ERA20C November snow index and DJF NAO for the period 1901-2010. 

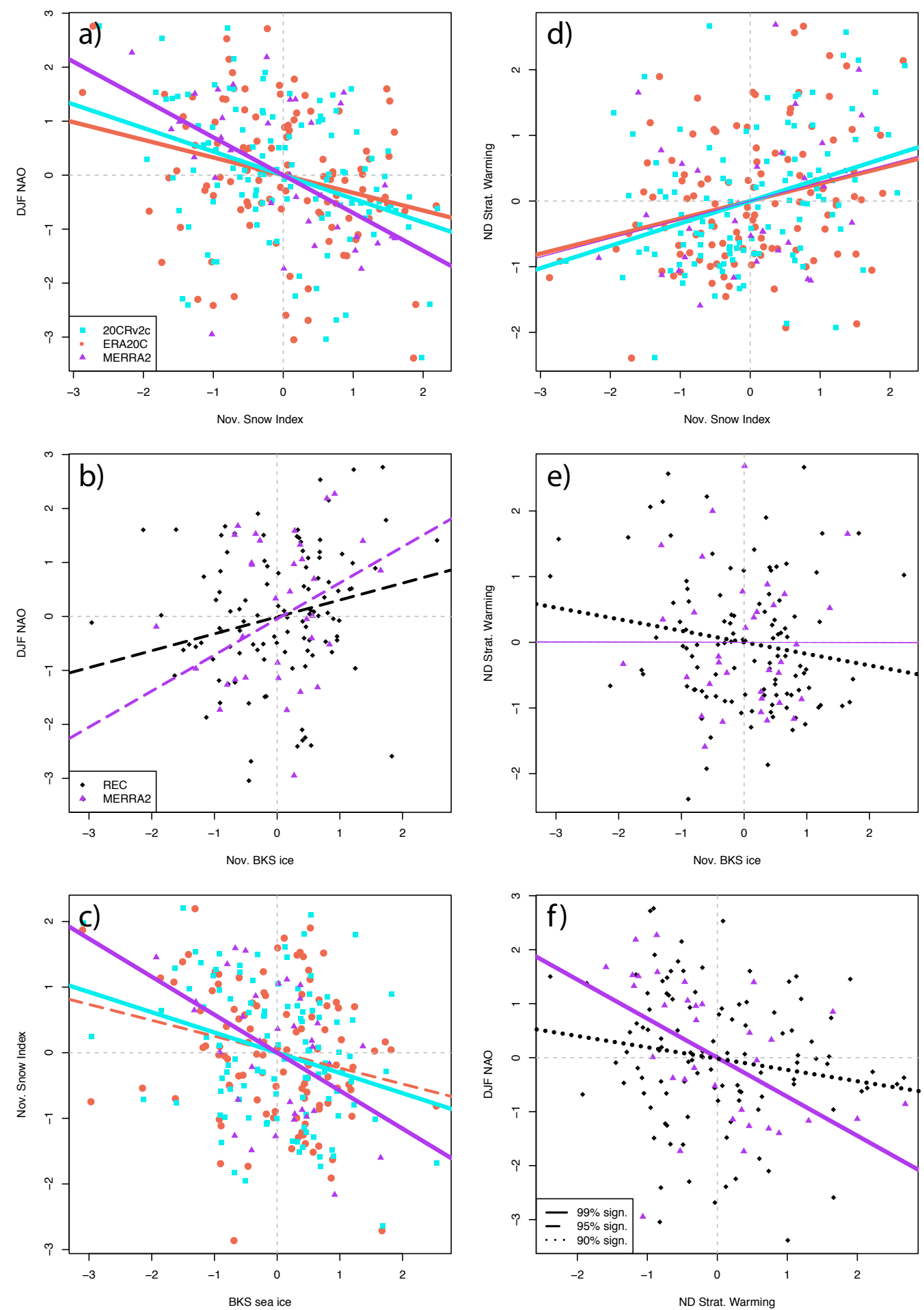

Figure 9: Linear Regression plots for selected variable pairs covering the period 1901-2010 in ERA20C, 20CRv2c as well as Reconstructions and 1981-2015 for MERRA2. a) November snow index versus DJF NAO, b) November BKS ice concentration vs DJF NAO, c) November BKS ice concentration vs November snow index, d) November snow index vs. mean November December polar $10 \mathrm{hPa} G P H$, e) November BKS ice concentration vs mean November December polar $10 \mathrm{hPa} G P H$ and f) mean November December polar $10 \mathrm{hPa} G P H$ vs DJF $N A O$ 

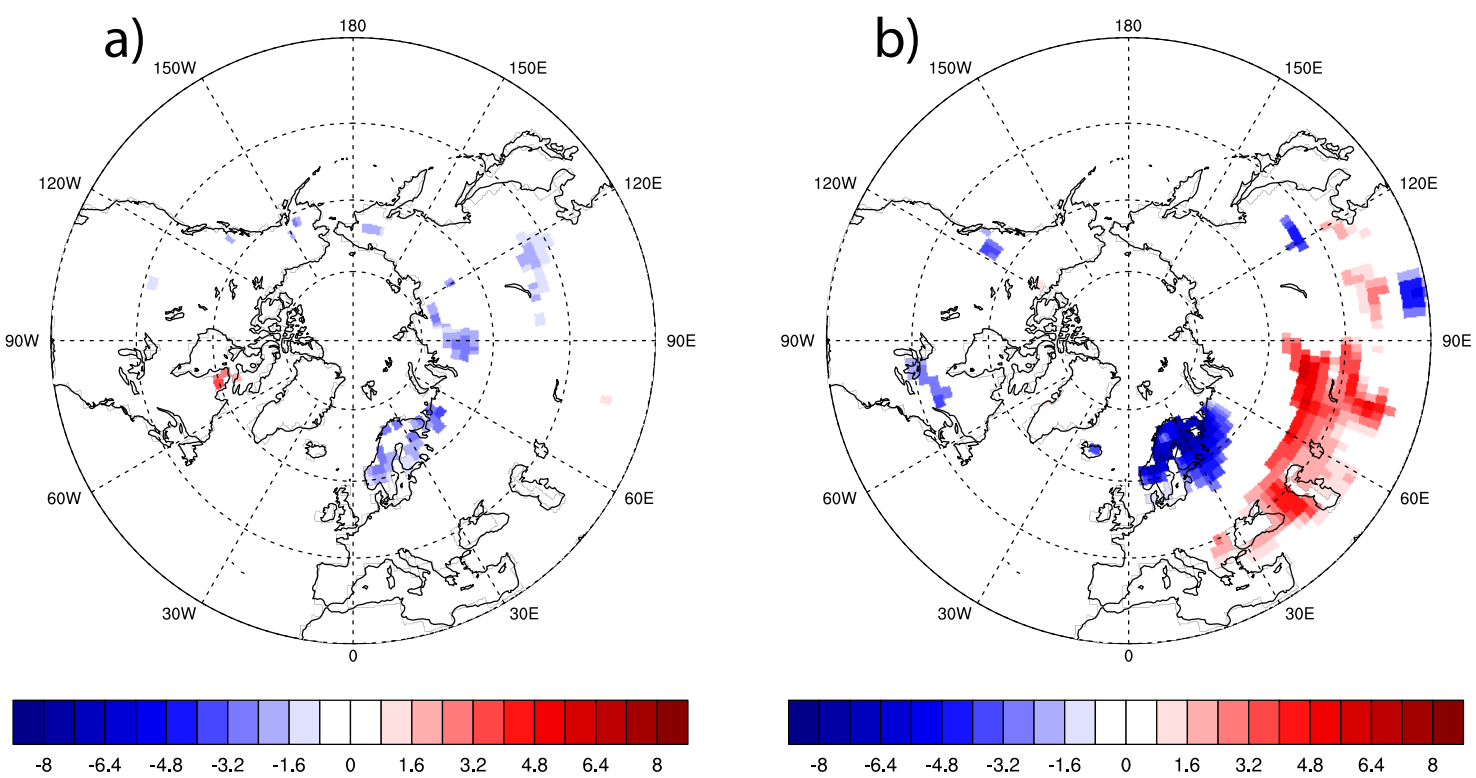

$[\% / s t d$ dev]

[\%/std dev]
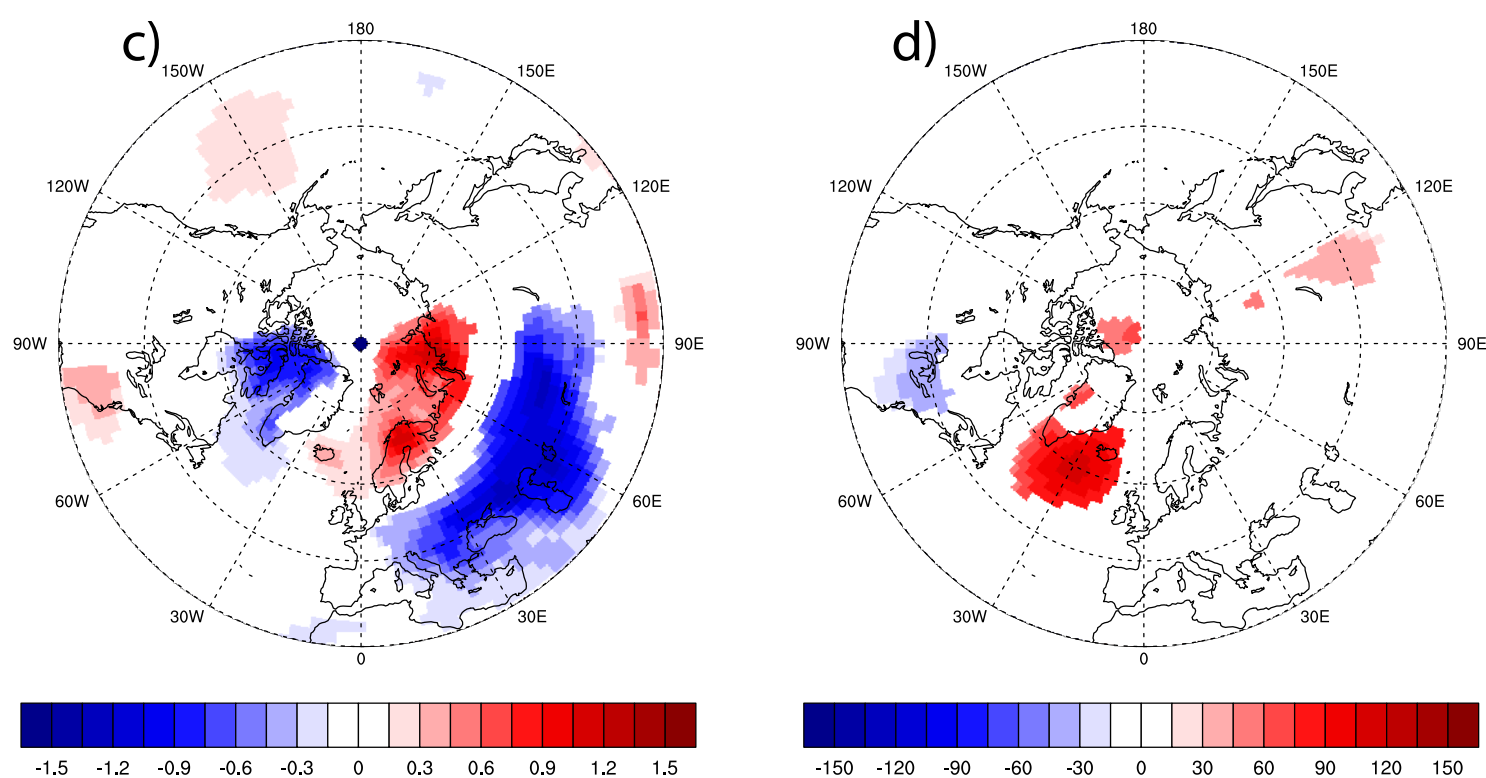

$[\mathrm{K} / \mathrm{std} \mathrm{dev}]$

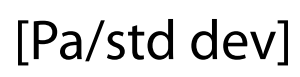

Figure 10: a) October snow cover anomalies [\%/std dev] projected onto the field averaged November blocking frequency, b) November snow cover anomalies [\%/std dev] projected onto the field averaged November blocking frequency, c) November $2 m$ temperature anomalies [K/std dev] projected onto the field averaged November blocking frequency and d) b) DJF SLP anomalies [Pa/std dev] projected onto the field averaged November blocking frequency. Field averaging was performed for the region, $\left(-10-80^{\circ} \mathrm{E}, 45-80^{\circ} \mathrm{N}\right)$ according to Peings 2019. 


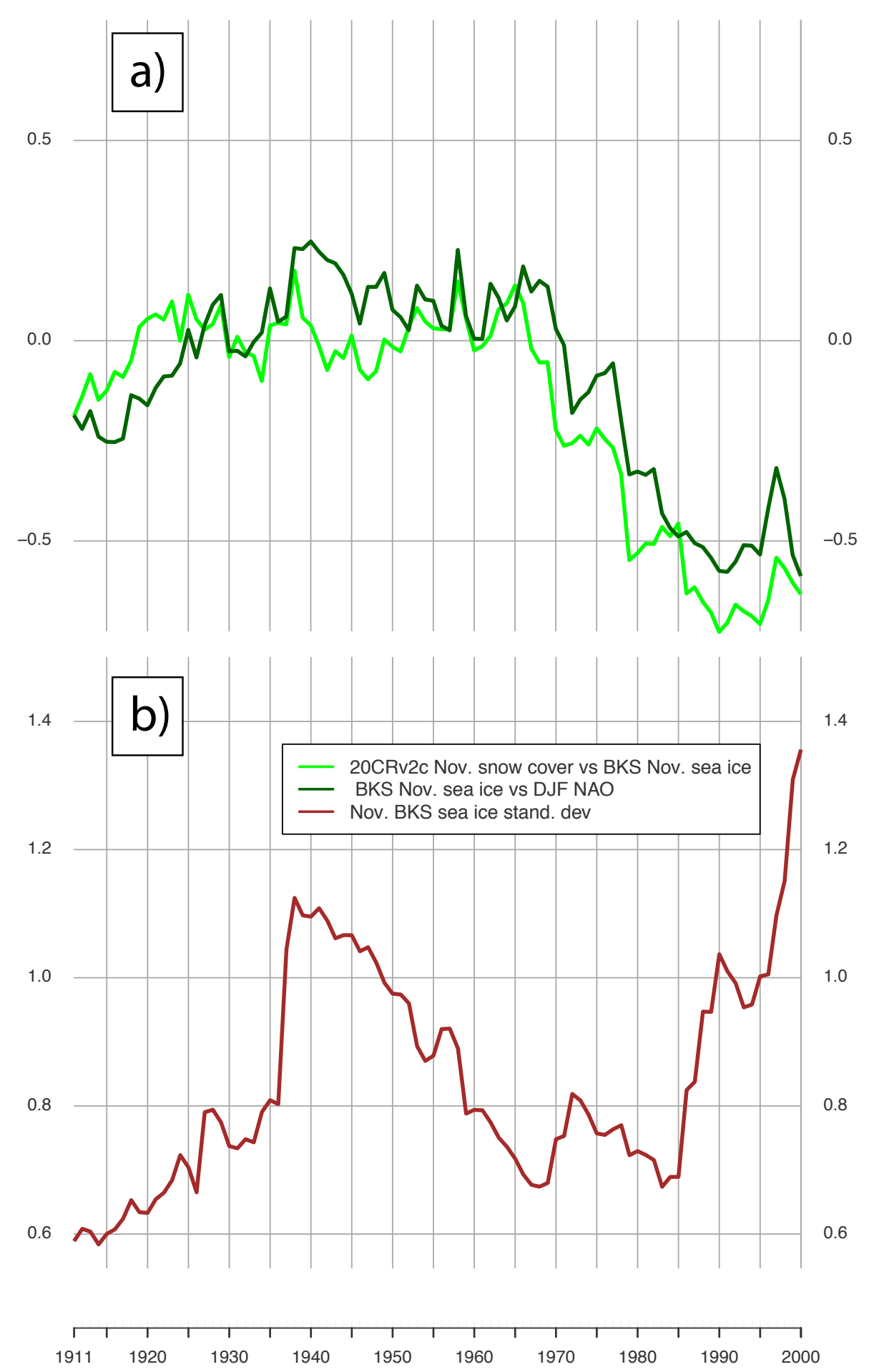

Figure 11: a) 21-year running correlation [R] between 20CRv2c November snow index and November BKS sea ice index as well as 21-year running correlation between November BKS sea ice index and DJF NAO index, b) 21-year running standard deviation for the November BKS sea ice index. 
Table 1: Durbin-Watson test statistics examining serial correlation for regression pairs in Figure 7.

\begin{tabular}{|c|c|c|c|}
\hline & ERA20C & 20CRv2c & Reconstruction \\
\hline $\begin{array}{l}\text { DJF NAO vs OCT SNOW } \\
\text { (Fig. 7a) }\end{array}$ & $\begin{array}{l}\mathrm{DW}=1.7336 \\
\text { p-value }=0.07846\end{array}$ & $\begin{array}{l}\mathrm{DW}=1.6661 \\
\mathrm{p}-\mathrm{value}=0.03779\end{array}$ & \\
\hline $\begin{array}{l}\text { DJF NAO vs NOV SNOW } \\
\text { (Fig. 7b) }\end{array}$ & $\begin{array}{l}\mathrm{DW}=1.6869, \\
\mathrm{p} \text {-value }=0.04772\end{array}$ & $\begin{array}{l}\mathrm{DW}=1.6915, \\
\mathrm{p} \text {-value }=0.05095\end{array}$ & \\
\hline $\begin{array}{l}\text { ND STRAT. WARMING } \\
\text { vs NOV SNOW (Fig. 7c) }\end{array}$ & $\begin{array}{l}\mathrm{DW}=2.2592 \\
\mathrm{p} \text {-value }=0.9145\end{array}$ & $\begin{array}{l}\mathrm{DW}=2.2009 \\
\mathrm{p} \text {-value }=0.8535\end{array}$ & \\
\hline $\begin{array}{l}\text { DJF NAO vs NOV. BKS } \\
\text { ice (Fig. 7e) }\end{array}$ & & & $\begin{array}{l}\mathrm{DW}=1.7831 \\
\mathrm{p} \text {-value }=0.1181\end{array}$ \\
\hline $\begin{array}{l}\text { ND STRAT. WARMING } \\
\text { vs NOV BKS ice }\end{array}$ & & & $\begin{array}{l}\mathrm{DW}=2.2879 \\
\mathrm{p} \text {-value }=0.9318\end{array}$ \\
\hline $\begin{array}{l}\text { NOV SNOW vs NOV BKS } \\
\text { ice (Fig. 7d) }\end{array}$ & $\begin{array}{l}\mathrm{DW}=1.8652 \\
\mathrm{p} \text {-value }=0.2267\end{array}$ & $\begin{array}{l}\mathrm{DW}=1.7097 \\
\mathrm{p} \text {-value }=0.05781\end{array}$ & \\
\hline $\begin{array}{l}\text { ND STRAT. WARMING } \\
\text { vs NDJF NAO (Fig. 7f) }\end{array}$ & & & $\begin{array}{l}\mathrm{DW}=1.6319 \\
\mathrm{p} \text {-value }=0.02664\end{array}$ \\
\hline
\end{tabular}

71 Table 2: Partial correlation and multiple regression for sea ice, PV ND and snow

\begin{tabular}{|l|l|l|l|l|l|l|}
\hline & Estimate & P-Value & Statistic & N & GP & Method \\
\hline $\begin{array}{l}\text { partial correlation } \\
\text { between NAO DJF and } \\
\text { 20CRv2c November } \\
\text { snow index given BKS } \\
\text { November sea ice index }\end{array}$ & -0.296866 & 0.0017207 & -3.215778 & 110 & 1 & Pearson \\
\hline $\begin{array}{l}\text { partial correlation } \\
\text { between NAO DJF and } \\
\text { ERA20C November } \\
\text { snow index given BKS } \\
\text { November sea ice index }\end{array}$ & -0.211391 & 0.0273459 & -2.237205 & 110 & 1 & Pearson \\
\hline $\begin{array}{l}\text { partial correlation } \\
\text { between NAO DJF and } \\
\text { BKS November sea ice } \\
\text { index given 20CRv2c } \\
\text { November snow index }\end{array}$ & 0.182415 & 0.0576334 & 1.919118 & 110 & 1 & Pearson \\
\hline $\begin{array}{l}\text { partial correlation } \\
\text { between NAO DJF and } \\
\text { BKS November sea ice } \\
\text { index given ERA20C } \\
\text { November snow index }\end{array}$ & 0.216711 & 0.02360975 & 2.296252 & 110 & 1 & Pearson \\
\hline $\begin{array}{l}\text { partial correlation } \\
\text { between NAO DJF and } \\
\text { ERA20C November } \\
\text { December mean polar 10 } \\
\text { hPa GPH given } \\
\text { 20CRv2c November } \\
\text { snow index }\end{array}$ & -0.041280 & 0.6699642 & -0.427376 & 110 & 1 & Pearson \\
\hline $\begin{array}{l}\text { partial correlation } \\
\text { between NAO DJF and } \\
\text { 20CRv2c November } \\
\text { snow index given } \\
\text { ERA20C November }\end{array}$ & -0.318160 & 0.00074774 & -3.471462 & 110 & 1 & Pearson \\
\hline
\end{tabular}


72

\begin{tabular}{|l|l|l|l|l|l|l|}
\hline $\begin{array}{l}\text { December mean polar 10 } \\
\text { hPa GPH }\end{array}$ & & & & & \\
\hline partial correlation & -0.274639 & 0.00402170 & -2.940663 & 110 & 2 & Pearson \\
between NAO DJF and \\
20CRv2c November \\
snow index given BKS \\
November sea ice index \\
and ERA20C November \\
December mean polar 10 & & & & & & \\
hPa GPH & & & & & & \\
\hline
\end{tabular}

73

74 